\title{
Estrategias de manejo de la milpa maya en Xoy, Peto, Yucatán
}

\section{Management strategies of the milpa maya in Xoy, Peto, Yucatán}

\section{Elia María Ku-Pech 1 (D), \\ Javier Orlando Mijangos- \\ Cortés ${ }^{1:}$ [0, \\ Rubén Humberto Andueza- \\ $\operatorname{Noh}^{2}$ (-), \\ Mariana Chávez-Pesqueira $\sqrt{\mathbb{0}}$ \\ Paulino Simá-Polanco ${ }^{1}$ \\ José Luis Simá-Gómez ${ }^{1}$ \\ Luis Manuel Arias-Reyes ${ }^{3}$}

${ }^{1}$ Centro de Investigación Científica de Yucatán, A.C. C. $43130 x$ 32 y 34, CP. 97205. Chuburná de Hidalgo, Mérida, Yucatán.

${ }^{2}$ CONACYT-Instituto Tecnológico de Conkal. Km 16.3 Antigua Carretera Mérida-Motul, CP. 97345. Conkal, Yucatán.

${ }^{3}$ Investigador

Calle 43 No.

Independiente.

Colonia Chuburná de Hidalgo, CP.

97205. Mérida, Yucatán, México.

*Autor de correspondencia: jomijangos@cicy.mx

\section{Nota científica}

Recibido: 16 de abril de 2019

Aceptado: 24 de septiembre de 2019

Como citar: Ku-Pech EM Mijangos-Cortés JO, AnduezaNoh RH, Chávez-Pesqueira M, Simá-Polanco P, Simá-Gómez JL, Arias-Reyes LM (2020) Estrategias de manejo de la milpa maya en Xoy, Peto, Yucatán. Ecosistemas y Recursos Agropecuarios 7(1): e2244. DOI: 10.19136/era.a7n1.2244
RESUMEN. El sistema tradicional milpa, manejado mayormente bajo temporal en Yucatán, México; es importante a nivel social, biológico y ambiental. La milpa ha perdurado a través del tiempo por los conocimientos ancestrales transmitidos por generaciones. Por lo anterior, el objetivo fue conocer las estrategias de manejo realizadas por los milperos en su actividad agrícola y la conservación de la diversidad biológica. La investigación se efectuó en la comunidad maya de Xoy, Peto, Yucatán aplicando encuestas semiestructuradas al $30 \%$ de la población ejidataria que siembra maíz. La diversidad de cultivos reportada en Xoy fue de 22 variedades nativas: ocho de maíz, siete de frijol lima, cuatro de calabaza y tres de frijol común. Se detectó que la familia es el determinante para la conservación de la diversidad de especies cultivadas en la milpa con una prioridad en la conservación de semillas nativas entre los milperos.

Palabras clave: Ambiental, nativa, semilla, social, tradicional.

ABSTRACT. The traditional crop system called milpa, managed mostly under temporary in Yucatan, Mexico it has been important at a social, biological and environmental level. The milpa has lasted through time by the ancestral knowledge transmitted from generations. From the above the objective was to know the management strategies carried out by the milperos in its agricultural activity and the conservation of the biological diversity. The research was carried out in the mayan community of Xoy, Peto, Yucatan applying semi-structured surveys to $30 \%$ of the ejidataria population that sows at least corn. The diversity of crops reported in Xoy was 22 native varieties: eight of corn, seven of lime bean, four of squash and three of common bean. It was detected that the family is the determinant for the conservation of the diversity of species grown in the milpa with a priority in the conservation of native seeds among the milperos.

Key words: Environmental, native, seed, social, traditional 


\section{INTRODUCCIÓN}

El manejo de la milpa maya tradicional involucra una diversidad de actividades y estrategias derivadas del conocimiento milenario de los milperos, que van desde la elección del terreno adecuado para el establecimiento de los cultivos hasta el consumo, almacenamiento de grano y selección de semilla (Salazar et al. 2016). En Yucatán, la milpa se maneja bajo temporal con marcada estacionalidad, una de lluvias y otra de sequías que hace que la siembra sólo pueda efectuarse una vez al año, siendo el número de hectáreas por milpero muy variable (Wommack 2018, Rodríguez y Arias 2014). Llegada la etapa de cosecha y de acuerdo a los métodos tradicionales de resguardo en las comunidades de Yucatán, la producción puede ser almacenada en trojes, estructuras elaboradas con madera de diferentes especies y ramas de huano (Sabal japa), por uno o dos años durante su uso (Lowe 2015, Cabrera 2014).

El manejo y la conservación de los recursos genéticos cultivados en la milpa está rodeada de múltiples factores, entre ellos el social, biológico y ambiental (CONABIO 2012). A través de múltiples generaciones el conocimiento que envuelve a la milpa se ha transmitido siendo un vínculo entre las generaciones pasadas y futuras. A lo largo del tiempo, la milpa ha perdurado hasta nuestros días, generando la conservación in situ de ciertas variedades de interés alimenticio para los milperos, mientras que en otras comunidades ha ido sufriendo adaptaciones de acuerdo al modus vivendi que predomina en la actualidad (Zizumbo y Colunga 2016). En el tema alimenticio el proceso de nixtamalización se hace presente, una técnica milenaria que ha coadyuvado en el consumo de maíces nativos en las comunidades y por ende a la conservación de la milpa (Vázquez et al. 2016). Por lo anterior, el objetivo del estudio fue conocer las estrategias de manejo desde la siembra hasta la cosecha, el almacenamiento y consumo realizadas por los milperos, la diversidad biológica actual bajo conservación en las milpas, los factores sociales y ambientales que giran en torno a la milpa, así como los cambios que ha sufrido con el tiempo y que determinan su actual situación.

\section{MATERIALES Y MÉTODOS}

\section{Área de estudio}

Al sur del estado se encuentra la comunidad de Xoy, del municipio de Peto, Yucatán, México ubicada a $20^{\circ} 08^{\prime} 03^{\prime \prime}$ LN y $88^{\circ} 58^{\prime} 02$ " LO, a una altura promedio de $35 \mathrm{msnm}$, con clima cálido subhúmedo, temperatura media anual de $26^{\circ} \mathrm{C}$ y precipitación pluvial media anual de $1050 \mathrm{~mm}$. Tiene una población total de 714 individuos, 353 hombres y 361 mujeres indígenas de los cuales el $99.30 \%$ de los hombres y el $48 \%$ de las mujeres tiene una escolaridad básica incompleta y el $14 \%$ no cuenta con escolaridad (CONEVAL 2010). En el 2017 se reportaban 162 ejidatarios de los que 121 se encuentran activos de acuerdo con datos proporcionados por el Comisario Ejidal.

\section{Investigación Etnobotánica}

Se aplicaron encuestas semiestructuradas a los ejidatarios milperos de Xoy en el 2017 para conocer las prácticas agrícolas efectuadas en la milpa, específicamente sobre las variedades de maíz, frijol y calabaza cultivadas. Se realizó un muestreo de conglomerados que abarcó los cuatro puntos cardinales de la comunidad y se eligieron aleatoriamente 48 ejidatarios milperos, que corresponden al $30 \%$ del total de ejidatarios. Este diseño de investigación tiene la ventaja de que cada unidad de población posee la misma posibilidad de formar parte de la muestra siendo representativa, además de que permite contar con un panorama general de la milpa que abarca a los diferentes grupos presentes en la comunidad de estudio.

En las encuestas se recabaron los datos generales del milpero y de la milpa como años de uso del suelo, apoyo en mano de obra y problemática de los cultivos en el ciclo agrícola 2016 y 2017, así como las variedades sembradas y razón de elección. También se indagó sobre los rendimientos obtenidos y destino de la producción. Con la finalidad de conocer el consumo del maíz, se encuestó a los responsables y a 10 usuarios del molino y la tortillería, indagando sobre la procedencia y el consumo de las variedades nativas por las familias milperas mediante el proceso de nixtamalización. En las encuestas 
semiestructuradas se utilizaron preguntas abiertas y cerradas. En el caso de las preguntas cerradas se tuvo de elección única, dicotómica y politómica. Los datos se analizaron con estadística descriptiva y de correlaciones de variables como integrantes de familia, edad del milpero, superficie para milpa, número de variedades sembradas en maíz y calabaza, entre otros, con el paquete estadístico SPSS Versión 19 (IBM 2010).

\section{RESULTADOS Y DISCUSIÓN}

Los ejidatarios encuestados han vivido muchos años en la comunidad de estudio, un $94 \%$ de ellos son originarios de la comunidad de Xoy, únicamente un $6 \%$ provienen de otras comunidades de Yucatán. Un $92 \%$ de la población encuestada fueron hombres y un $8 \%$ mujeres, todos inmersos en la actividad milpera, de los cuales, el $78 \%$ depende exclusivamente de la milpa para satisfacer sus necesidades de subsistencia.

\section{Diversidad de cultivos}

La diversidad encontrada en las milpas de Xoy fue de 22 variedades nativas, ocho corresponden a maíz, siete a frijol lima, cuatro a calabaza y tres a frijol común. Los milperos en Xoy tuvieron de forma simultánea hasta cinco variedades nativas de maíz en el 2016, pero en el ciclo 2017 este número se redujo a cuatro, disminuyendo también en frijol de dos a uno y la calabaza de tres a dos. Para el maíz los milperos manejaron al menos una variedad, no así para frijol común y frijol lima donde pueden prescindir de su siembra, lo que refleja la ausencia de las leguminosas en algunas milpas, debido principalmente a la alta mano de obra que demanda el cultivo de frijol.

Las variedades preferidas por los milperos en la comunidad de Xoy se debe a preferencias como el rendimiento, precocidad y tolerancia al estrés hídrico. En maíz la variedad Nal Xoy y la raza Xnuk Nal fueron de las más reportadas, debido al alto rendimiento propio de estos maíces. El maíz Nal Tel también fue uno de los preferidos debido a su precocidad, lo que ha permitido la conservación de esta raza en la comunidad. Por el contrario, la raza Dzit Bacal apare- ció escasamente en las preferencias de los milperos de Xoy. Al respecto Uzcanga et al. (2017) reportaron la preferencia de Dzit Bacal entre agricultores de la península de Yucatán, debido a características sobresalientes como tolerancia a la sequía intraestival, adaptación a suelos pedregosos y satisfacción de exigencias organolépticas con productos como tortilla, atole, pozol, entre otros. La entrada de maíces mejorados se hizo presente en Xoy, debido a la introducción de materiales mejorados a través de los programas de gobierno en las comunidades mayas, pero el uso y preferencia hacia estos maíces se ha visto limitado por el hecho de tener que invertir en la adquisición de semilla en cada ciclo agrícola, lo que implica un gasto (Flores y García 2016, Mijangos 2018).

En frijol Xcoli Bu'ul y Tsama' fueron de las variedades preferidas por los milperos en Xoy. Para el frijol lima la variedad Xmejen fue preferida por su precocidad en producción y la Xnuk por generar granos de mayor tamaño. Lo que coincide con Mariaca (2015) quien reporta las mismas variedades en la milpa maya yucateca. Para la calabaza las variedades más reportadas en las milpas de Xoy fueron C. moschata (Xmejen K'um, Xnuk K'um) y $C$. argyrosperma (Xtop), y en un porcentaje sumamente bajo la variedad C. pepo (Tz'ol). Al respecto Canul et al. (2005) en la región centro-oriente de Yucatán reportaron sólo a estas dos primeras especies; siendo más frecuentes las variedades Xnuk k'um e Xmejen K'um, dentro de la especie C. moschata, como resultado de la predilección por sus características organolépticas.

\section{Relación de los factores sociales y ambientales con la diversidad en las milpas}

La edad del milpero y la familia son determinantes para la continuidad de las actividades de la milpa. La mayoría de las personas que se dedican actualmente a la milpa son de edad avanzada (58\%), esto es, personas que rebasan los 60 años, siendo la edad máxima reportada de 90 y la edad media de 42 años. A mayor edad se vuelve más complicado el manejo y cuidado de la milpa, observándose que los milperos que superan los 70 años de edad lle- 
gan a abandonar por completo la actividad, lo que lleva a un alto riesgo de pérdida de las especies si no se continúa por los hijos o nietos la siembra de la milpa. Al respecto, Salazar et al. (2016) reportan que la edad del productor tiene relación directa con la diversidad agrícola, debido a que, con el paso del tiempo, el conocimiento adquirido por el milpero valora el manejo de un mayor número de cultivos con el objetivo de garantizar la cosecha. Las variables relacionadas de forma positiva con la diversidad en las milpas en Xoy fueron el porcentaje de consumo familiar, el consumo por animales y la venta de la producción, no así la edad del milpero (Tabla 1). La importancia derivada de poseer animales de traspatio por las familias milperas también favorece la diversidad de fauna de aves, ganado porcino y vacuno, ayudando así a la conservación de especies vegetales y animales. La variable plagas y enfermedades estuvo correlacionada de forma positiva $(r=0.340)$ con el número de variedades sembradas de maíz ( $r=$ 0.358). Lo mismo sucede con la variable sequía que estuvo relacionada de forma positiva $(r=0.277)$ con la diversidad de calabaza, debido al conocimiento que el milpero tiene sobre el comportamiento de este cultivo ante la escasez de agua. Se encontró que el tamaño de la familia y el consumo que se tenga de las especies y variedades tiene una relación directa con el número de las variedades sembradas en la milpa.

\section{Asociación de especies y variedades}

Del total de la población encuestada, el $73 \%$ mencionó el abandono de siembra de alguna especie y/o variedad, que puede ir desde uno hasta cinco por milpero en variedades de maíz, frijol lima y común, así como de calabaza. Algunas de las razones de abandono de siembra por milperos fueron ausencia de lluvias, altos costos de mano de obra sobre todo para Phaseolus y el desgaste de los suelos que no ofrecen óptimos rendimientos. En Xoy el $89 \%$ de los encuestados brinda un uso continuo a los suelos de la milpa, sin otorgar el periodo de barbecho o descanso. Las milpas son sembradas de forma continua hasta por 70 años, con valor promedio de 21 años. Un barbecho en las milpas de entre siete a 15 años

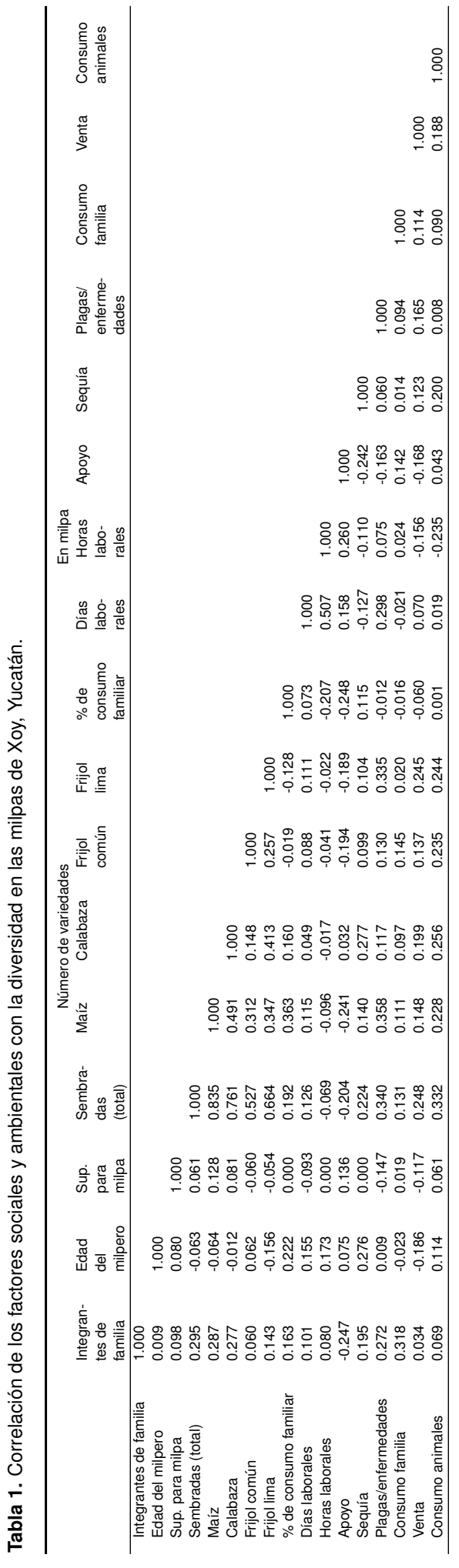


es necesario para recuperar la fertilidad de suelos tipo kankab que tiene Xoy (Rodríguez y Arias 2014). Sin embargo, el $77 \%$ de los milperos se establecen a largo plazo en un sitio de trabajo y difícilmente se trasladan a un área nueva. La razón se debe a que las tierras para la milpa son ejidales, pudiendo ser ocupadas por otros milperos en el momento en que son abandonadas, además de la ausencia de nuevos espacios adecuados para la milpa, como suelos tipo kankab (cambisol y luvisol) que son ligeramente más profundos y menos pedregosos.

\section{Selección de semillas}

La actividad de selección permite a los milperos preservar las mejores características en las especies de interés para siembra, fijando de esta manera aspectos sobresalientes que el milpero haya detectado en sus variedades. El momento de selección por los milperos es justo después de la cosecha $(60 \%)$, previo al inicio de siembra del siguiente ciclo productivo (23\%), durante el almacenamiento (12\%) y durante el consumo (5\%). El tamaño del fruto y semillas son los mejores caracteres a fijar para la siguiente generación. El 67\% de los encuestados no cuenta con el apoyo de algún miembro de la familia para las actividades de selección. Considerando que el productor sea de edad avanzada y que sea el único que realiza la actividad de selección, la permanencia y transmisión del conocimiento para esta actividad en particular podría estar en grave riesgo en Xoy, con la posibilidad de pérdida del germoplasma que se cultiva en la milpa.

\section{Siembra}

El principal factor que determina la siembra en las milpas son las precipitaciones irregulares, adaptándose el milpero a estos cambios ambientales (Ortiz et al. 2014). El mes de siembra en Xoy es en junio, de acuerdo a las primeras precipitaciones, sin embargo, algunos milperos optan por sembrar en mayo, siembra denominada "tikim muk" que en lengua maya significa sembrar en seco debido a la falta de humedad en los suelos. El $100 \%$ de los encuestados siembran maíz, el $77 \%$ calabaza, el 55\% frijol lima y el $36 \%$ frijol común. La siembra de fri- jol en las milpas de Xoy, por lo general se efectúa en un espacio reducido dentro de la milpa o incluso puede prescindirse de este cultivo. Por lo que la asociación de maíz, frijol y calabaza simultáneamente en la milpa, en la actualidad, es menor pudiendo ser un monocultivo de maíz. No obstante, el término milpa sigue estando arraigado en la población de Xoy por aspectos hereditarios, denominando milpa al espacio donde se encuentra presente el maíz, principal cultivo destinado a su alimentación.

\section{Cosecha}

El milpero puede preferir realizar una sola recolecta, o bien realizar varias cosechas en diferentes momentos dependiendo de la variedad, comenzando por aquéllas precoces. La fecha de la cosecha en Xoy está definida por las necesidades y preferencias alimenticias del milpero y su familia. En la comunidad de Xoy, los rendimientos de maíz en las milpas son bajos, con valores mínimos de 20 $\mathrm{kg} \mathrm{ha}^{-1} \mathrm{y}$ un valor promedio de $670 \mathrm{~kg} \mathrm{ha}^{-1}$, donde la producción no logra satisfacer las demandas del mercado tanto nacional como regional e incluso local. En maíz los rendimientos no superaron el valor de $1 \mathrm{t} \mathrm{ha}^{-1}$, para la mayoría de las variedades sembradas durante el ciclo agrícola 2016. A excepción de algunas variedades como Nal Xoy, reportado por algunos milperos. En frijol lima, la variedad Xmejen superó $1 \mathrm{t} \mathrm{ha}{ }^{-1}$ de rendimiento. En frijol común las únicas dos variedades que superaron el rendimiento de $100 \mathrm{~kg} \mathrm{ha}^{-1}$ fueron Xcoli Bu'ul y Xmejen Bu'ul. En calabaza, la mayoría de los milperos obtuvieron rendimientos menores a $500 \mathrm{~kg} \mathrm{ha}^{-1}$ durante el ciclo agrícola 2016. La única variedad que superó los 500 $\mathrm{kg} \mathrm{ha}^{-1}$ fue Xtop (C. argyrosperma).

\section{Destino de la producción}

La cosecha obtenida en las milpas se dirige principalmente para el autoconsumo, predominando por sobre los demás destinos: consumo de la familia $(30 \%)$, semilla (29\%), alimentación de los animales de traspatio (27\%) y venta (14\%). A finales del siglo $\mathrm{XX}$ con la introducción de pesticidas y semillas mejoradas en las milpas se incrementaron los costos de producción, se dio la caída de los precios del maíz y 
se redujeron las superficies de siembra llegando a ser sólo para autoconsumo. Al igual, hay relación directa entre el tamaño de la unidad familiar, la cantidad de monte que se tumbaba para hacer milpa (2 a 6 ha), y la participación de la familia (dos a seis adultos), lo que facilitaba que una extensión extra se siembre para la venta o la engorda de cerdos, de esa manera se pueden obtener ingresos monetarios derivados de la milpa (Pérez 2013).

En maíz se destina en promedio $4.57 \mathrm{~kg}$ diarios para el consumo de una familia, por lo que al año se consumen $1668.05 \mathrm{~kg}$. Considerando que se obtienen $670 \mathrm{~kg} \mathrm{ha}^{-1}$ en promedio de maíz, la cantidad requerida para consumo indica un déficit en producción. Por lo que un milpero con 3 ha de siembra obtendría un excedente de $341.95 \mathrm{~kg}$ que puede destinar para reservorio de semilla, alimentación de los animales de traspatio y venta. Es importante considerar que no todos los milperos manejan 3 ha de forma simultánea, por lo que los rendimientos que logren alcanzar y las cantidades para los diferentes destinos fluctúa de un milpero a otro.

La producción de maíz obtenida por los milperos de Xoy solo les permite almacenar un promedio de $638 \mathrm{~kg}$ de maíz, siendo los frijoles los más afectados con una mínima cantidad (16 a 19 $\mathrm{kg}$ ). Para el almacenamiento se requiere de un espacio físico como la troje, el interior de la vivienda del milpero u otro sitio destinado, de acuerdo a las circunstancias. En Xoy el $48 \%$ de los milperos eligen la casa como sitio de almacén y muy por debajo, el almacenamiento en trojes ubicadas en la milpa. Los milperos que no cuentan con troje lo asocian a la baja productividad obtenida en la milpa, al no haber un excedente en la producción no se requiere de una estructura especial de almacenamiento. Por otra parte, el esfuerzo físico que implica el corte de huano y de las maderas para la elaboración de las trojes, donde en muchos casos no se cuenta con el apoyo de otros integrantes de la familia, complica la labor para los milperos de edad avanzada. Otro sitio de almacenamiento es el refrigerador, resultado de los nuevos conocimientos sobre temas de conservación entre los milperos y la adaptación a los cambios en el tiempo, donde las bajas temperaturas permiten un almacenaje de semillas por periodos prolongados. EI tiempo de almacenaje, independientemente del sitio, para maíz, calabaza, frijol común y frijol lima fue de siete, seis, 10 y 12 meses en promedio, respectivamente, por lo que el maíz no abastece durante todo el año a los milperos de Xoy para suplir sus necesidades recurren a la compra de maíz.

\section{Procesamiento para el consumo de maíz}

En Xoy las mujeres se dedican a la elaboración de alimentos provenientes de la milpa, donde el proceso de nixtamalizado permite la transformación de maíz a masa, proceso que no varía mucho del que se efectuaba en tiempos prehispánicos (Meléndez et al. 2014). El molino, como se le conoce coloquialmente en Yucatán, y la tortillería son los establecimientos que ayudan a las mujeres a agilizar el proceso de elaboración de alimentos. Los maíces destinados a molienda mayormente son de color blanco y amarillo, y en menor medida los granos pigmentados como el maíz morado y rojo. La obtención de materia prima se basa en la compra de maíz nativo a algunos productores de la comunidad que cuentan con excedente de producción, sin embargo, las cantidades son mínimas por lo que se acude a expendios como DICONSA (programa de abasto rural que opera a través de tiendas comunitarias). El $90 \%$ de los encuestados hacen uso de este tipo de maíz, dado que la producción obtenida en la milpa no cubre las necesidades alimenticias de las familias.

La cantidad destinada para molienda se basa en el número de comidas, el tipo de comida que se realiza en el hogar, el número de integrantes por familia y el número de animales de traspatio con los que se cuentan. Debido a la escasez en la producción, la alimentación se complementa con harinas procesadas, afectando de forma indirecta la comercialización, preferencia de maíces nativos y el mantenimiento de la milpa, situación que se presenta en la mayoría de las comunidades rurales de la Península de Yucatán, prevaleciendo el control que tiene esta marca en la cadena agroalimentaria en México, contradiciendo el tema de autosuficiencia alimentaria y de reactivación del sector agrícola (Soria y Palacio 2014). 
Considerar a la milpa como un sistema multifactorial con cambios en el tiempo favorece su comprensión ante la complejidad que representa. EI saber milenario que poseen los milperos puede estar en riesgo de pérdida, así como la transmisión del conocimiento ancestral que envuelve la conservación de germoplasma de las especies alimenticias, debido a que más de la mitad de los milperos entrevistados realizan las actividades de la milpa de manera soli- taria y son personas de edad avanzada. Xoy, Yucatán es un sitio interesante de estudio, por contar con una alta diversidad de cultivos en la milpa, además de ser referente para actividades de conservación de la raza de maíz Nal Tel, al ser el maíz preferido para siembra por los milperos, con una predominancia de los maíces nativos por encima de los maíces mejorados en la milpa.

\section{LITERATURA CITADA}

Cabrera PA (2014) Estrategias de sustentabilidad en el solar maya Yucateco en Mérida, México. GeoGraphos 5: 1-32.

Canul KJ, Ramírez PV, Castillo FG, Chávez JL (2005) Diversidad morfológica de calabaza cultivada en el centrooriente de Yucatán, México. Revista Fitotecnia Mexicana 28: 339-349.

CONABIO (2012) La Milpa. Portal Biodiversidad Mexicana. https://www.biodiversidad.gob.mx/usos/alimentacion/ milpa.html. Fecha de consulta: 1 de abril de 2019.

CONEVAL (2010) Informe anual sobre la situación de pobreza y rezago social. Peto, Yucatán. Subsecretaría de Prospectiva, Planeación y Evaluación. SEDESOL. https://www.gob.mx/cms/uploads/attachment/file/47191/ Yucatan_058.pdf. Fecha de consulta: 12 de agosto de 2019.

Flores CL, García SJ (2016) Beneficios de la adopción de semilla mejorada de maíz en la región central de Puebla. Revista Fitotecnia Mexicana 39: 277-283.

IBM (2010) IBM SPSS Statistics for Windows, Versión 19.0. Armonk, NY: IBM Corp.

Lowe LS (2015) Reseñas Fabianne de Pierrebourg y Mario Humberto Ruz (coords.), Nah, otoch. Concepción, factura y atributos de la morada maya. Estudios de cultura maya 45: 267-270.

Mariaca MR (2015) La milpa maya yucateca en el siglo XVI: Evidencias etnohistóricas y conjeturas. Etnobiología 13: 1-38.

Meléndez PC, Quintero RA, Ruiz GM, Camacho DA (2014) Del maíz a la tortilla: nixtamalización. Synthesis Digital 1: 36-38.

Mijangos CJ (2018) Colecta de maíces nativos en regiones estratégicas de la Península de Yucatán. Versión 1.5. Comisión nacional para el conocimiento y uso de la biodiversidad. https://www.gbif.org/dataset/8da53f1a8ed7-4c91-8b47-f51f3be8bd1d. Fecha de consulta: 3 de marzo de 2019.

Ortiz TJ, Sánchez SO, Ramos PJ (2014) Actividades productivas y manejo de la milpa en tres comunidades campesinas del municipio de Jesús Carranza, Veracruz, México. Polibotánica 38: 173-191.

Pérez RM (2013) Efraím H. Xolocotzi. Contribuciones al estudio de las familias mayas milperas. Etnobiología 11: 14-27.

Rodríguez A, Arias RL (2014) La milpa y el maizal: retos al desarrollo rural en México y Perú. Etnobiología 12: 76-89.

Salazar BL, Magaña MM, Aguilar JA, Ricalde PM (2016) Factores socioeconómicos asociados al aprovechamiento de la agrobiodiversidad de la milpa en Yucatán. Ecosistemas y Recursos Agropecuarios 3: 391-400. 
Soria SG, Palacio VM (2014) El escenario actual de la alimentación en México. Textos \& Contextos 13: 128-142.

Uzcanga PN, Larqué SB, Del Angel PA, Rangel FM, Cano GA (2017) Preferencias de los agricultores por semillas mejoradas y nativas de maíz en la Península de Yucatán, México. Revista Mexicana de Ciencias Agrícolas 8: 1021-1033.

Vázquez GV, Castañeda SM, Cárcamo TN, Santos TA (2016) Género y medio ambiente en México: una antología. Primera edición. Universidad Nacional Autónoma de México, Centro Regional de Investigaciones Multidisciplinarias. Cuernavaca, Morelos, México. 306p.

Wommack WB (2018) Arquitectos paisajistas mayas y estética territorial en la Península de Yucatán. Gremium 5: 25-34.

Zizumbo VD, Colunga GP (2016) El uso de las cenizas como posible precursor de la nixtamalización en el oeste de Mesoamérica. Revista de Geografía Agrícola 57: 7-18. 